\title{
On the Influence of Manufacturing Strategy of 3D-Printed Polymer Substrates on Cold Spray Deposition
}

Antonio Viscusi, Antonello Astarita, Domenico Borrelli, Antonio Caraviello, Luigi Carrino, Roberta Della Gatta, Valentina Lopresto, Ilaria Papa, Alessia Serena Perna, Raffaele Sansone and Antonino Squillace

Antonio Viscusi. Department of Chemical, Materials and Production Engineering, University of Naples Federico II, Piazzale V.

Tecchio 80, 80125 Napoli, Italy.

Corresponding author: antonio.viscusi@unina.it

Antonello Astarita. Department of Chemical, Materials and Production Engineering, University of Naples Federico II, Piazzale V.

Tecchio 80, 80125 Napoli, Italy.

Domenico Borrelli. Sòphia High Tech, Via Romani 228, 80048, Sant'Anastasia NA, Italy.

Antonio Caraviello. Sòphia High Tech, Via Romani 228, 80048, Sant'Anastasia NA, Italy.

Luigi Carrino. Department of Chemical, Materials and Production Engineering, University of Naples Federico II, Piazzale V. Tecchio 80, 80125 Napoli, Italy.

Roberta Della Gatta. Department of Chemical, Materials and Production Engineering, University of Naples Federico II, Piazzale V. Tecchio 80, 80125 Napoli, Italy.

Valentina Lopresto. Department of Chemical, Materials and Production Engineering, University of Naples Federico II, Piazzale V. Tecchio 80, 80125 Napoli, Italy.

Ilaria Papa. Department of Chemical, Materials and Production Engineering, University of Naples Federico II, Piazzale V. Tecchio 80, 80125 Napoli, Italy.

Alessia Serena Perna. Department of Chemical, Materials and Production Engineering, University of Naples Federico II, Piazzale V. Tecchio 80, 80125 Napoli, Italy.

University of Bergamo, Bergamo, Italy.

Raffaele Sansone. Sòphia High Tech, Via Romani 228, 80048, Sant'Anastasia NA, Italy.

Antonino Squillace. Department of Chemical, Materials and Production Engineering, University of Naples Federico II, Piazzale V.

Tecchio 80, 80125 Napoli, Italy.

Abstract. PMCs are anisotropic and heterogeneous structures with excellent performances in terms of mechanical strength and stiffness, coupled with reduced weight, widely used in engineering sectors. The use of PMCs can be further extended by improving their surface properties such as electrical conductivity, erosion, radiation and lightning protection. In this context, the surface metallization seems to be best solution. In particular, the cold spray (CS) technique candidates as a potential method for the manufacturing of a metal coating on PMCs' surface. However, the design and the manufacturing methods of PMCs can play a crucial role for an effective metallization through CS. The additive manufacturing technologies for composite materials can be used to manufacture customized reinforced polymer-based panels, like PMCs; the most common method for printing them is the Fused Filament Fabrication (FFF) technique which relies on the thermal extrusion of a thermoplastic feedstock from a mobile heated nozzle. Therefore, this research activity aims to manufacture customized PMCs panels by using FFF technology for the substrate and the cold spray technique for the metallization in order to study the influence of the substrate manufacturing strategy on CS deposition process. For this purpose, three kind of 3D-printed PMCs were manufactured through the FFF technology by varying the percentage fill of the Onyx polymeric matrix and aluminum powders were sprayed on the substrates with a low-pressure cold spray (LPCS) system; both FFF and CS process parameters were varied to study the process in its wholeness. Microscope analyses were carried out to analyze the influence of the manufacturing strategy on the coating quality.

Keywords. 3D-Printing, PMCs, Cold Spray, Manufacturing strategy, FFF Technology, Metal Deposition 
On the Influence of Manufacturing Strategy of 3D-Printed Polymer Substrates on Cold Spr...

\section{Introduction}

The reinforced polymer matrix composites (PMCs) are the most used in engineering fields due to their lightness and high mechanical properties. These noticeable characteristics made PMCs very attractive in a wide variety of industries: from sporting goods to civil construction to aerospace [1].

The applications of the composite materials in engineering fields could be widely extended if some of the surface properties of PMCs were enhanced; for instance, it would be very interesting the manufacturing of a metallic layer on the surface of PMCs in order to modify their surface properties such as electrical conductivity, thermal conductivity, electromagnetic shielding, erosion, radiation and lightning protection, so resulting in a novel and more functional composite structure. This idea would be possible through the metallization process of PMCs.

To date, there exist different techniques for the metallization of polymer-based materials. Unfortunately, these techniques have disadvantages that strongly limit their uses and applications, as reported by Zhou et al. [2]. Moreover, some of these technologies may lead the polymeric substrate to deterioration, making the metallization impossible to carry out [3]. In this context, the cold gas dynamic spray technology (generally referred as cold spray), as a "cold" thermal spray coating technique, was proved to be the best solution for PMCs metallization [4]. The relatively low temperature and pressure values of the gas involved do not lead to the deterioration of the substrate so resulting in an effective technique for the manufacturing of a metallic layer on the polymer-based materials, like PMCs.

Unlike in thermal spraying and fusion-based additive manufacturing technologies, during CS, materials are deposited by kinetic energy instead of thermal energy; hence, the problems associated with material melting and solidification are avoided. The micron-sized particles (10-100 $\mu \mathrm{m}$ in diameter [5]) are dragged by the carrier gas (air, nitrogen, helium) which expands at supersonic conditions by means of a converging-diverging de-Laval nozzle. The achieved impact velocities usually range between 300 and $1200 \mathrm{~m} \mathrm{~s}-1$ [6]. The result is that the particles impact on the substrate in the solid solid-state at relatively high velocity, they deform and bond together for the coating formation and grow-up [7].

The study of metal deposition though CS on metallic substrates was carried out extensively in the last decades [8]. However, the attentions of the researches in the last years are moving toward the cold spray deposition on polymeric substrates [9]; it was shown that CS deposition mechanisms and the resultant quality of PMCs metallization are dependent on CS process parameters such as the gas species, the gas pressure and temperature, the standoff distance and the powder typology [10]. It is known, that the deposition process can be effectively carried out if the abovementioned process parameters are properly set. For instance, pressure values lower than 10 bar are generally used to perform the metallization of polymers [11]. Moreover, soft and low melting temperature metals such as tin, zinc and aluminum were proved to be ease to spray on PMCs or to be used as intermediate layer to produce a coating build-up [2]. A typical successful case is the LPCS metallization of a PVC substrate using a tin undercoating that enabled a generation of copper coating $800 \mu \mathrm{m}$ thick [12]. Viscusi et al. [13] proposed an original manufacturing method that provides the surface thermoplastic polylactic acid (PLA) treatment for thermosetting basalt fiber-reinforced plastics, so overcoming the issues pointed out by Ganesan et al. [12] for the metallization of thermoset-based materials.

Together, these polymers metallization studies have shown a good feasibility of the cold spray additive manufacturing, also proving the influence of the substrate typology and conditions on the CS deposition process; that means that the design and the manufacturing methods of PMCs may play a crucial role for an effective metallization through CS, as reported by the authors [14]. Hence, it appears evident that the tailoring of PMCs to be used as CSed substrates would provide the best solution for studying in more details the influence of the manufacturing process of the polymer substrates on the coating deposition. In other words, the polymer-based substrates may be designed and realized "ad hoc" for analyze the effects of the composite stratification, lay-up sequence and typology on the cold spray impact process. 
Unfortunately, the traditional manufacturing techniques for composite materials require expensive equipment and increase manufacturing costs; however, the last researches proved that the additive manufacturing techniques for the polymer-based materials seem to be able to produce highly customizable parts, e.g. rapid prototyping, personalized devices or structures with complex geometry [15]. The most common method for printing a thermoplastic polymer is the Fused Filament Fabrication method which relies on the thermal extrusion of a thermoplastic feedstock from a mobile heated nozzle [16].

In this method, the polymer filament (ABS, PLA, PC, PEEK and Nylon [17]) is extruded through the nozzle that traces the part's cross sectional geometry layer-by-layer. The nozzle contains resistive heaters that keep the polymer at a temperature just above its melting point, so that it flows easily through the nozzle and forms the layer. Reinforcing materials, such as short and/or continuous fibers, are added into the polymer matrix during printing, in order to produce a composite structure which typically exhibits improved mechanical properties [16].

On the base of these premises, it is evident that FFF technology represents the best solution for the production of customized PMCs samples to be integrated with the CS technique for the metallization process. Therefore, this research activity aims to propose an innovative manufacturing route for the production of tailored PMCs panels by using the FFF technology for the substrate and the cold spray technology for the metallic coating in order to study the influence of the substrate manufacturing strategy on CS deposition process. For this purpose, three kind of 3D-printed PMCs were manufactured through the FFF technology by varying the filling percentage of the Onyx polymeric matrix; in particular, filler percentages of $30 \%, 40 \%$ and $50 \%$ were considered in this activity.

The samples were metallized through the LPCS technology by setting proper CS process parameters. Micron-sized aluminum powders were used for PMCs metallization. The products were observed through SEM and confocal microscopies aiming to analyze the influence of the filler percentage, namely the resulting surface texturing, on the coating deposition and the metallization quality.

\section{Materials and methods}

The 3D-printed panels were manufactured through the Markforged X7 facility based on FFF-printing process. The last is based on the melting of the plastic filament by means of a heated extrusion head and its application in a single layer to the platform. Each additional layer is applied with the previous links (since it is melted) and, after cooling, the material hardens for the manufacturing of the final products (Fig. 1). The printer facilities that use the FFF technology are usually based on a cartesian coordinate system: the extruder can move on three perpendicular mutual axes (x,y,z). The melting extrusion head firstly moves in x-y plane to print a given layer and then it moves vertically (along $\mathrm{z}$ ) for the deposition of the following layers. The deposition process is repeated, layer-by-layer, for the printing of the designed object [18]. The Markforged technology allows for set the scan patter of the nozzle, namely the filling strategy, for the printing of the 3D-component. 


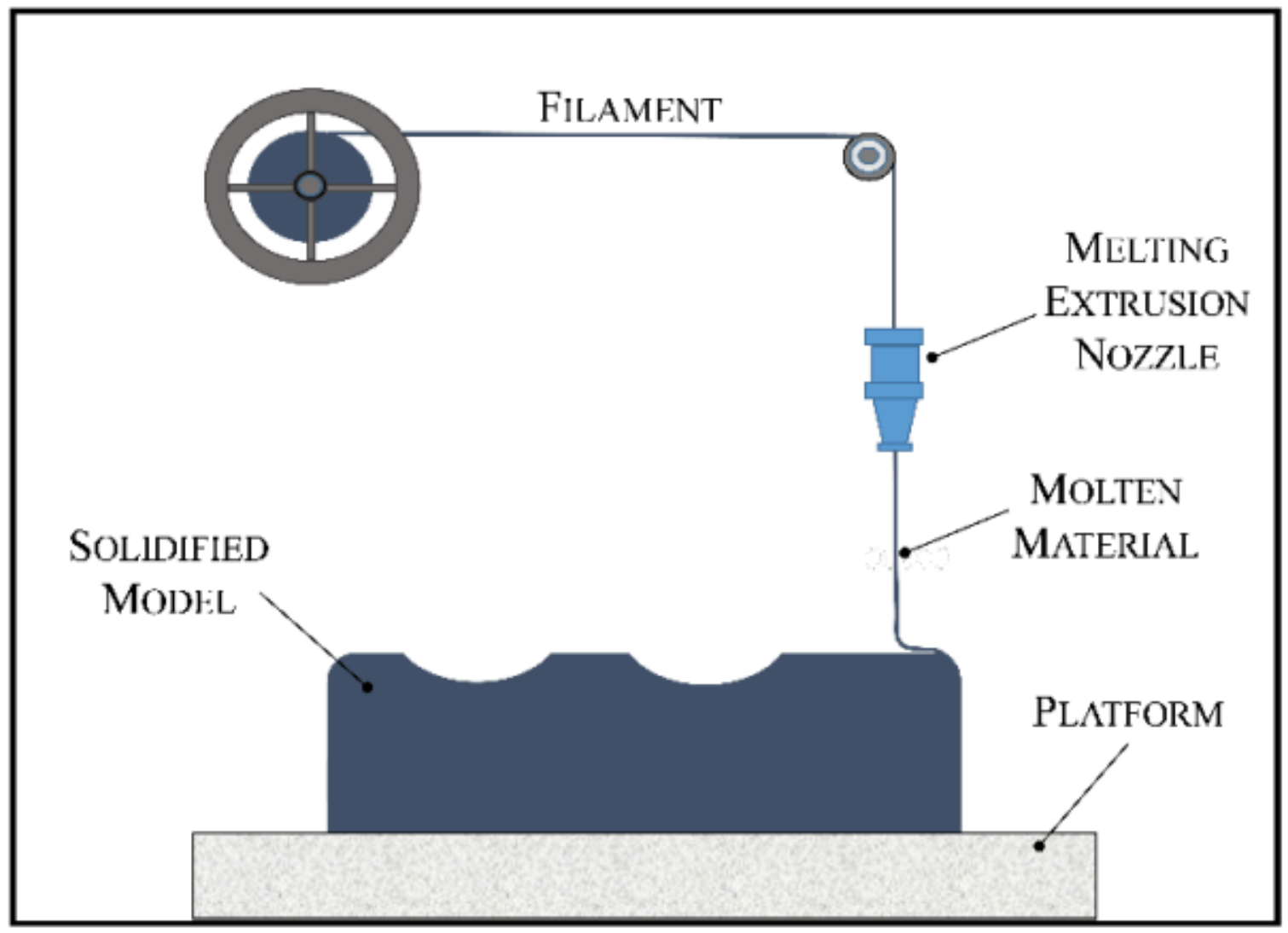

Fig. 1. Simple sketch of FFF process.

The thermoplastic material that was used in this activity is Onyx made of nylon mixed with short carbon fibers. It is stronger, harder and more resistant to heat than other plastic 3D-printing materials. The material provides toughness of nylon with added stiffness and strength provided by the fibers dispersed inside, heat resistance up to $145^{\circ} \mathrm{C}$ and a high resistance to adverse conditions [19].

The manufacturing of the specimens used in this research was performed at different percentages filling of the printed components in order to analyze the influence of the resulting surface texturing on CS deposition process. In particular, triangular filling structures were used for each specimen and three different percentages filling values (30\%, 40\% and $50 \%$ ) were considered for the samples and denoted below as T30, T40 and T50, respectively. An exemplificative sketch of the scan pattern used is reported in Fig. 2. It is worth noting that firstly the nozzle defines the boundaries of each layer (the red pattern performed in the x-y plane in Fig. 2) and then the triangular filler for the matrix core structure. Moreover, the top and the bottom surfaces are always printed with one layer of $100 \%$ fill Onyx filament (the so called in-fill solid strategy at $45^{\circ}[20]$ ) to take advantage of the higher surface quality finishing. 


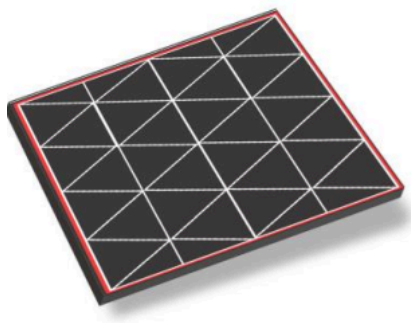

a)

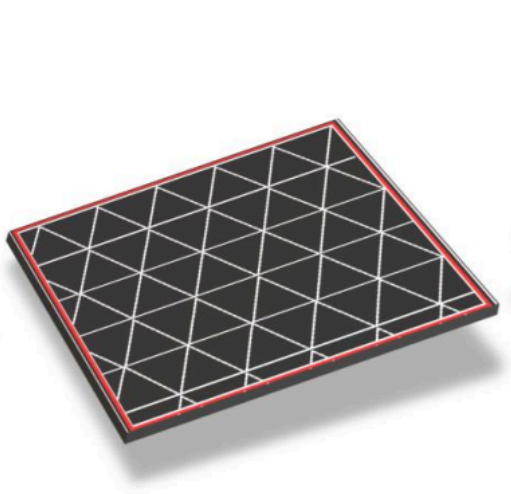

b)

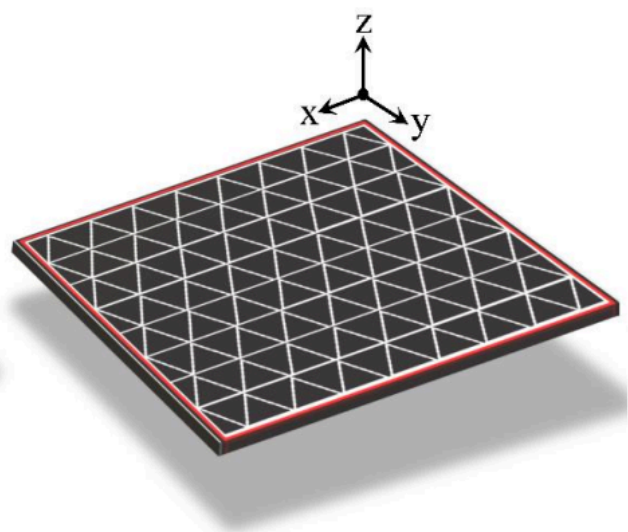

c)

Fig. 2. Illustrative sketch of triangular filling structures for three different percentages filling values: T30 (a), T40 (b), T50 (c).

The square-shaped panels $(50 \times 50 \mathrm{~mm})$ have a thickness of about $2.0 \mathrm{~mm}$. Each layer constituting the stratified panel is about $0.125 \mathrm{~mm}$ depending on the heated nozzle geometry. Moreover, printing is done at $260{ }^{\circ} \mathrm{C}$ with an estimated speed of $6.90 \mathrm{~cm}^{3} / \mathrm{h}$.

Dymet 423 equipment was used for surface metallization. It is a low-pressure cold spray facility with a spray gun, two powder feeders and a control unit. Control unit includes fluid pressure and fluid temperature control and powder feeders switch with powder feed rate regulators. The spray gun was attached to a robot (HIGH-Z S-400/T CNC-Technik) to allow for control and repeatability of the coating deposition. Compressed air was used as carrier gas as a consequence of the low velocities required when spraying on polymeric substrates [3]. Micron sized powders of aluminum alloy AlSi10Mg, provided by LPW South Europe, were chosen with a particle mean size of about $25 \mu \mathrm{m}$.

The range of suitable CS process parameters was determined through preliminary experimental tests, which are not here reported for the sake of brevity, as well as literature results. In fact, different coating tracks with a travel speed of the spraying gun equal to $1 \mathrm{~mm} / \mathrm{s}$ were preliminarily produced by varying the process parameters in a wide range (inlet gas temperature: $150-600{ }^{\circ} \mathrm{C}$, inlet gas pressure: $4-8$ bar, stand-off distance (SoD): $10-80 \mathrm{~mm}$ ). In detail, a single-track coating was developed on the surface of each laminate by spraying only one layer of aluminum particles. The inlet gas pressure and temperature were set to 6 bar and $300^{\circ} \mathrm{C}$, respectively, the SoD was set to $45 \mathrm{~mm}$ and kept constant during the deposition.

Confocal microscope observations (Leica DCM3D Scan) were carried out on a $5 \times 5 \mathrm{~mm}$ coated area of each specimen; moreover, SEM analyses were carried out by using a Hitachi TM 3000 SEM aiming to analyze the influence of the substrate characteristics on the CS deposition process.

\section{Results and discussion}

The samples realized through the integrated manufacturing process involving FFF technology for the polymer-based substrates and the CS technique for the metal deposition are shown in Fig. 3. From a quick visual inspection, it is possible to notice the effectiveness of the process developed by using the abovementioned process parameters for both the substrate and coating's manufacturing. The coating seems to be almost continuous with an effective deposition of the particles. The resulting surface texturing from the three different percentages filling values is well visible and its influence on the coating deposition will be investigated in details below. 


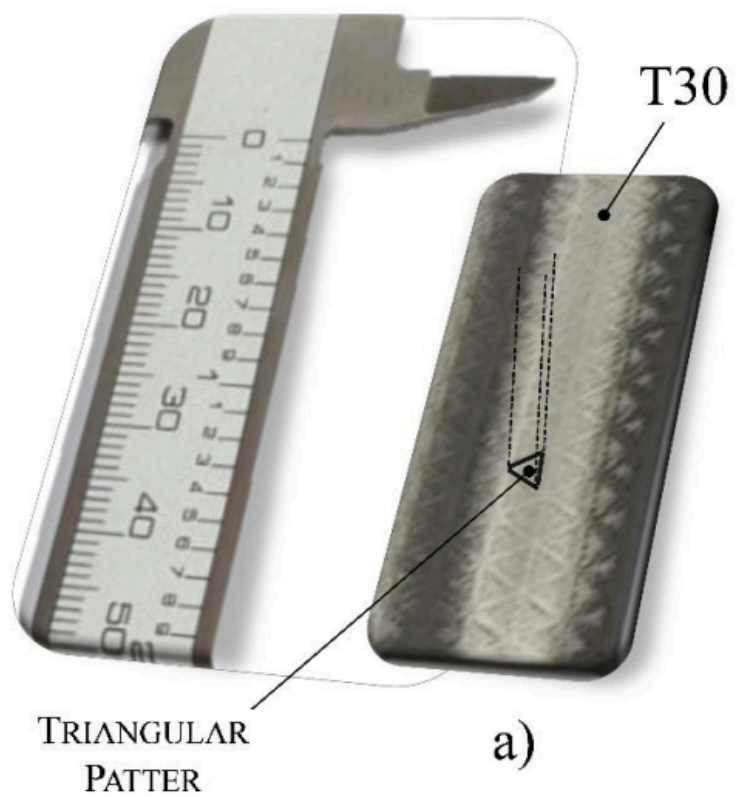

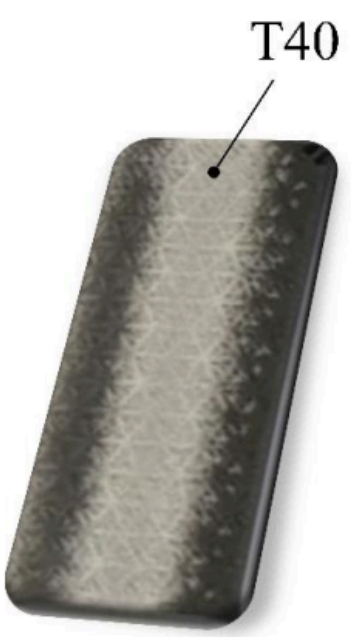

b)

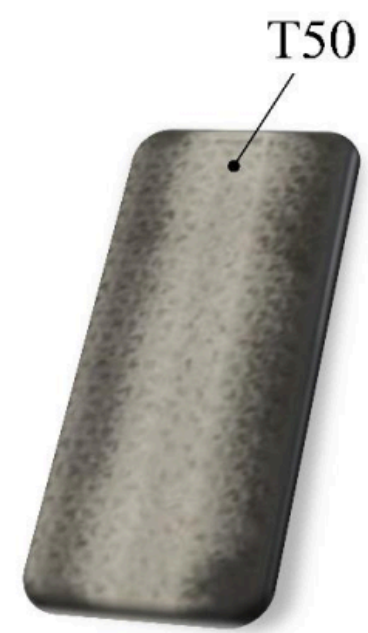

c)

Fig. 3. Real pictures of the coated samples highlighting the manufacturing strategy adopted: T30 (a), T40 (b), T50 (c).

The results from the confocal microscope analyses carried out on a square-coated area of each sample typology (T30, T40 and T50) are reported in Fig. 4. Both the 3D-reconstruction of the surface undulation along with the representative roughness profile for each panel are reported in the figure. It can be seen from the surface undulation pictures that the triangular scan pattern is well marked after the metal coating deposition with the colored blue zones which point out the filling strategy. Moreover, the dimensions of the highlighted triangular structures tend to be smaller with the increasing of the filling percentage, the like-grid surface of the T50 specimens is denser than the others (T30 and T40) with a reduced space between two consecutive valleys. This is due to the one-single pass CS deposition that does not cover entirely the surface morphology; this was done to emphasize the effect of the scan pattern on CS impact mechanisms.
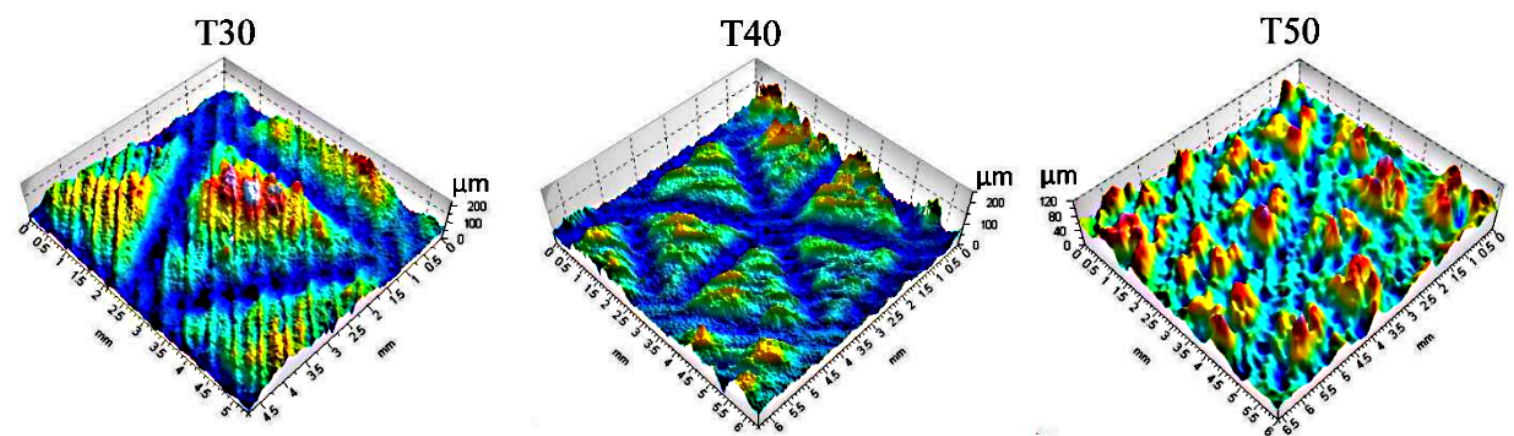

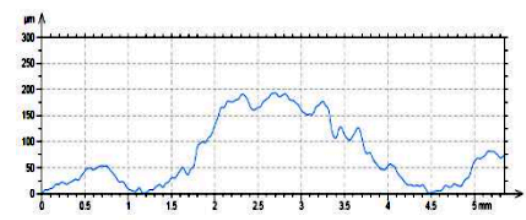

a)

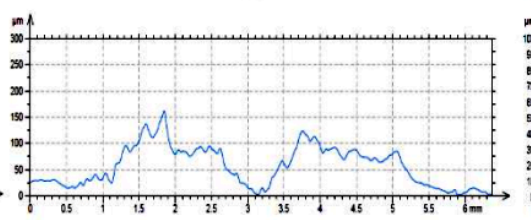

b)

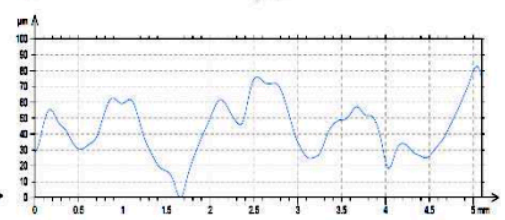

c)

Fig. 4. 3D-Reconstruction of the surface undulation along with the representative roughness profile for each substrate typology: T30 (a), T40 (b), T50 (c). 
By looking Fig. 4a, where the phenomena are more pronounced, the particles seem to be entirely impacted within each valley tracked by two consecutive polymer tracks reaching a coating heigh of about $200 \mu \mathrm{m}$. Moving toward Figs. $4 \mathrm{~b}$ and $4 \mathrm{c}$, it can be seen that the distances between two consecutive polymer tracks reduces with the impacting powders that tend to feel the presence of the surface texturing which promotes the deformation and the sticking of the particles $[21,22]$. In fact, due to the more deformation of them, the coating height tend to reduce of about $30 \%$ for T40 samples and $60 \%$ for T50 panels (compared to T30).

The effects of the filling strategy, namely of the resulting surface texturing on the deformation mechanisms of the particles, are also confirmed by SEM images (Fig. 5).

$\mathrm{T} 30$

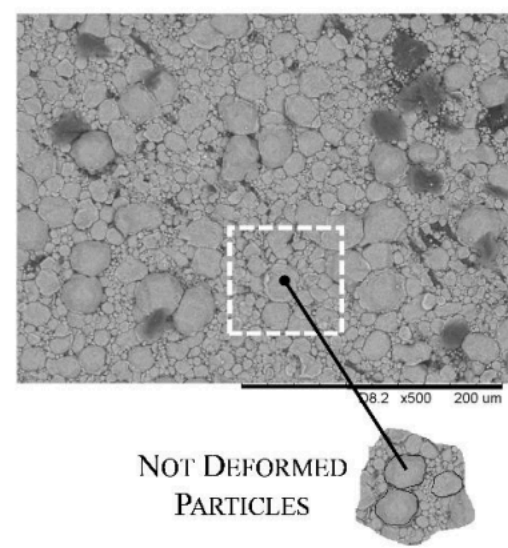

a)
T40

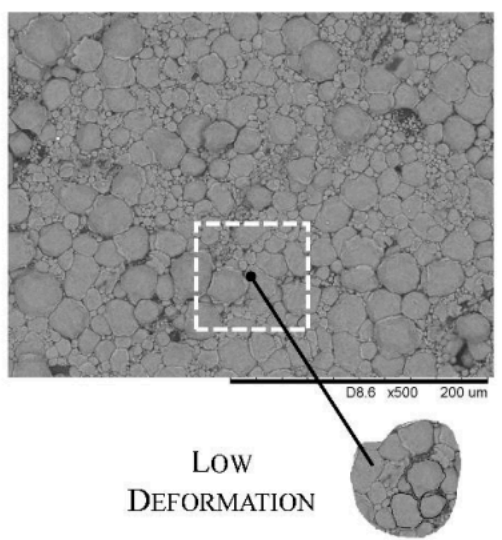

b)
$\mathrm{T} 50$

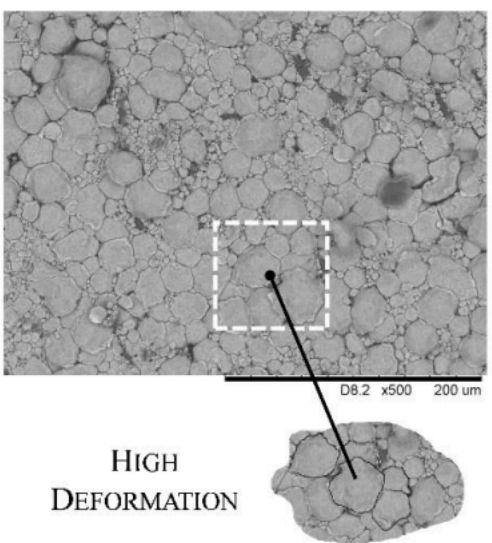

c)

Fig. 5. SEM pictures showing the particle deformation for each substrate typology: T30 (a), T40 (b), T50 (c).

It can be seen that when the substrate is characterized by relatively large "isolated dimples" (see T30 samples) the deformation and the sticking of the particles are not promoted (Fig. 5a). When the spacing of the like-grid surface's lines reduces (see T40 and T50 samples), with the valleys of the texturing that show a comparable dimension to the feedstock powder diameter, the particles strongly deform (Figs. $5 \mathrm{~b}$ and $5 \mathrm{c}$ ), flatten and become entangled between each valley, so promoting the particle interlocking mechanisms. In fact, from literature [9] it is known that the anchorage energy between the first layer and the polymeric substrate is smaller than the energy required by the second layer to adhere on the first one. The result is that the upcoming particles possessing a relatively high impact energy destroy the first layer making it impossible the coating grow-up. The results found in this research activity proved that the manufacturing strategy of the panels can have an influence on the surface texturing that, in turn, can have beneficial effects on the particles deformation mechanisms. In particular, when the filament filling is set to $50 \%$, the deformation of the particles is encouraged and the bonding mechanisms between the first layer and the substrate seem to be promoted. That means that is very difficult for the upcoming particles to remove them as they remain firmly anchored to the polymer surface.

\section{Conclusions}

The aim of this work was to manufacture customized PMCs panels by using the FFF technology for the substrates and the cold spray technique for the metallization in order to study the influence of the substrate manufacturing strategy on CS deposition process. For this purpose, three different percentages filling values (30\%, 40\% and 50\%) were considered for the samples production and the CS deposition was carried out. Therefore, on the basis of the experimental results discussed in the previous sections, the following conclusions can be drawn: 
On the Influence of Manufacturing Strategy of 3D-Printed Polymer Substrates on Cold Spr...

- FFF technology was proved to be a potential solution to produce customized 3D-printed PMCs panels by varying the filling percentage.

- The cold spray process was proved to be an effective technique to metallize 3D-printed substrates when the process parameters are properly set

- The effects of the filling strategy, namely of the resulting surface texturing on the deposition mechanisms, were proved; in particular, when the spacing of the like-grid surface's lines reduces (see T50 samples), the particles strongly deform, flatten and become entangled between each valley, so promoting the particle interlocking mechanisms. That means that when the filament filling strategy is set to $50 \%$, the particles seem to be better anchored to the polymeric substrates, so promoting the coating formation and grow-up.

\section{Bibliography}

[1] Zhang, X. Chen, Y. Hu, J. Recent advances in the development of aerospace materials. Progress in Aerospace Sciences, 2018, 97, 22-34.

[2] Zhou, X. L. Chen, A. F. Liu, J. C. Wu, X. K. Zhang, J. S. Preparation of metallic coatings on polymer matrix composites by cold spray. Surface and Coatings Technology, 2011, 206, 132-136.

[3] Lupoi, R. O’Neill, W. Deposition of metallic coatings on polymer surfaces using cold spray. Surface and Coatings Technology, 2010, 205, 2167-2173.

[4] Rokni, M. R. Feng, P. Widener, C. A. Nutt, S. R. Depositing Al-Based Metallic Coatings onto Polymer Substrates by Cold Spray. Journal of Thermal Spray Technology, 2019, 28, 1699-1708.

[5] Schmidt, T. Assadi, H. Gärtner, F. Richter, H. Stoltenhoff, T. Kreye, H. Klassen, T. From particle acceleration to impact and bonding in cold spraying. Journal of Thermal Spray Technology, 2009, 18, 794-808.

[6] Raletz, F. Vardelle, M. Ezo'o, G. Critical particle velocity under cold spray conditions. Surface and Coatings Technology, 2006, 201, 1942-1947.

[7] Assadi, H. Gärtner, F. Stoltenhoff, T. Kreye, H. Bonding mechanism in cold gas spraying. Acta Materialia, 2003, 51, 4379-4394.

[8] Viscusi, A. Astarita, A. Gatta, R. Della Rubino, F. A perspective review on the bonding mechanisms in cold gas dynamic spray. Surface Engineering, 2019, 35, 743-771.

[9] Che, H. Chu, X. Vo, P. Yue, S. Metallization of Various Polymers by Cold Spray. Journal of Thermal Spray Technology, 2018, 27, 169-178.

[10] Gonzalez, R. Ashrafizadeh, H. Lopera, A. Mertiny, P. McDonald, A. A Review of Thermal Spray Metallization of Polymer-Based Structures. Journal of Thermal Spray Technology, 2016, 25, 897-919.

[11] Raoelison, R. N. Xie, Y. Sapanathan, T. Planche, M. P. Kromer, R. Costil, S. Langlade, C. Cold gas dynamic spray technology: A comprehensive review of processing conditions for various technological developments till to date. Additive Manufacturing, 2018, 19, 134-159.

[12] Ganesan, A. Yamada, M. Fukumoto, M. Cold Spray Coating Deposition Mechanism on the Thermoplastic and Thermosetting Polymer Substrates. Journal of Thermal Spray Technology, 2013, 22, 1275-1282. 
[13] Viscusi, A. Antonucci, V. Carrino, L. Della Gatta, R. Lopresto, V. Papa, I. Perna, A. S. Ricciardi, M. R. Astarita, A. Manufacturing of an innovative composite structure: Design, manufacturing and impact behaviour. Composite Structures, 2020, 250, 112637.

[14] Della Gatta, R. Viscusi, A. Perna, A. S. Caraviello, A. Astarita, A. Cold spray process for the production of AlSi10Mg coatings on glass fibers reinforced polymers. Materials and Manufacturing Processes, 2020, 1-16.

[15] Parandoush, P. Lin, D. A review on additive manufacturing of polymer-fiber composites. Composite Structures, $2017,182,36-53$.

[16] Brenken, B. Barocio, E. Favaloro, A. Kunc, V. Pipes, R. B. Fused filament fabrication of fiber-reinforced polymers: A review. Additive Manufacturing, 2018, 21, 1-16.

[17] Popescu, D. Zapciu, A. Amza, C. Baciu, F. Marinescu, R. FDM process parameters influence over the mechanical properties of polymer specimens: A review. Polymer Testing, 2018, 69, 157-166.

[18] Rinaldi, M. Ghidini, T. Cecchini, F. Brandao, A. Nanni, F. Additive layer manufacturing of poly (ether ether ketone) via FDM. Composites Part B: Engineering, 2018, 145, 162-172.

[19] Kabir, S. M. F. Mathur, K. Seyam, A.-F. M. A critical review on 3D printed continuous fiber-reinforced composites: History, mechanism, materials and properties. Composite Structures, 2020, 232, 111476.

[20] Blok, L. G. Longana, M. L. Yu, H. Woods, B. K. S. An investigation into 3D printing of fibre reinforced thermoplastic composites. Additive Manufacturing, 2018, 22, 176-186.

[21] Kromer, R. Danlos, Y. Aubignat, E. Verdy, C. Costil, S. Coating deposition and adhesion enhancements by laser surface texturing-metallic particles on different classes of substrates in cold spraying process. Materials and Manufacturing Processes, 2017, 32, 1642-1652.

[22] Viscusi, A. Astarita, A. Genna, S. Leone, C. On the influence of different superficial laser texturing on the deposition of powders through cold spray process. Transactions of the Institute of Metal Finishing, 2018, 96, 34-40.

PDF automatically generated on 2021-05-19 21:16:24

Article url: https://popups.uliege.be/esaform21/index.php?id=3003

published by ULiège Library in Open Access under the terms and conditions of the CC-BY License (https://creativecommons.org/licenses/by/4.0) 